\title{
Extramedullary myeloid tumour (EMMT) of the gallbladder
} H Ojima, T Hasegawa, Y Matsuno, M Sakamoto

This report describes a rare case of an extramedullary myeloid tumour (EMMT) of the gallbladder in a patient without leukaemia. A 33 year old man visited a local hospital because of jaundice. Abdominal computed tomography revealed a tumorous mass measuring $6.0 \times 4.5 \mathrm{~cm}$ and involving the entire gallbladder. A percutaneous needle biopsy was attempted, but because adenocarcinoma could not be completely ruled out, the use of undue force was considered dangerous. Under a preoperative diagnosis of gallbladder carcinoma, a hepatopancreatoduodenectomy was performed. The tumour cells exhibited various amounts of eosinophilic cytoplasm, had medium sized round nuclei with indentation and grooving, and were strongly immunoreactive for myeloperoxidase, CD43, and c-kit protein (CD1 17). After surgery, the patient underwent combination chemotherapy as prescribed for cases of acute myeloblastic leukaemia. The patient did not develop acute leukaemia during a follow up period of four years. In conclusion, a correct diagnosis of EMMT can be made using appropriate immunohistochemical staining.

E xtramedullary myeloid tumour (EMMT), otherwise termed granulocytic sarcoma or chloroma, is a rare extramedullary tumour composed of immature cells of the myelomonocytic series. Males and females are equally affected, with a mean age of 48 years (range, 2-81). ${ }^{1}$ About $70 \%$ of reported cases are in patients with acute myelogenous leukaemia, chronic myelogenous leukaemia, or other myeloproliferative diseases, but in the remaining 30\% no known underlying disease has been noted at the time of diagnosis. The most common sites of involvement are the skin, lymph nodes, and bone, although other organs have been implicated. A large proportion (75-86\%) of EMMTs in nonleukaemic patients are initially misdiagnosed. ${ }^{2}$ An EMMT developing in the gallbladder of a patient without leukaemia is extremely rare. Geddy and Wedgwood reported a case of myelofibrosis of the gallbladder, ${ }^{3}$ but unlike our case, lesions outside the gallbladder were recognised in the bone marrow. Here, we report a rare case of EMMT of the gallbladder, detailing the clinicopathological and immunohistochemical features of this entity, which was accurately diagnosed and has been followed up for a long period.

\section{"A large proportion (75-86\%) of extramedullary myeloid tumours in non-leukaemic patients are initially mis- diagnosed"}

\section{CASE REPORT}

A 33 year old man visited a local hospital because of jaundice. He was diagnosed as having gallbladder carcinoma based on a radiographic examination, and was referred to the National Cancer Centre, Tokyo, Japan. Laboratory data indicated that carcinoembryonic antigen, CA19-9, and elastase concentrations were within normal limits, and that T-bilirubin ( $51 \mathrm{mg} /$ litre), D-bilirubin (37 mg/litre), alkaline phosphatase (688 IU/litre), glutamic oxaloacetic transaminase (84 U/ litre), glutamic pyruvate transaminase (317 U/litre), lactate dehydrogenase (431U/litre), and the white blood cell count $\left(9.6 \times 10^{9}\right.$ /litre $)$ were slightly raised.

Abdominal computed tomography imaging showed partial infiltration of the tumour into the gallbladder wall. We tried to perform a percutaneous needle biopsy, but because adenocarcinoma could not be completely ruled out the use of undue force was considered dangerous. We performed a cytological examination which was unable to provide definitive information on the lesion. The preoperative diagnosis was a malignant neoplasm that probably originated from the neck of the gallbladder, cystic duct, or common bile duct. However, it is unusual for a carcinoma to grow so large without showing signs of invasion of the liver and portal vein (fig 1A). The differential diagnosis was an extranodal malignant lymphoma, and a hepatopancreatoduodenectomy was performed.

Macroscopically, the gallbladder lumen was filled with blood and degenerative tissue, and the cut surface of the tumour had a nodular, well circumscribed, glistening appearance (fig 1B). The tumour measured $6.0 \times 4.5 \mathrm{~cm}$ at its maximum diameter.

Microscopically, the tumour cells had various amounts of eosinophilic cytoplasm and medium sized round nuclei with indentation and grooving. They were arranged in a trabecular to sheet-like pattern within the thin fibrous septa (fig 2A), and did not show a cohesive growth pattern. The tumour cells had invaded the muscular layer of the gallbladder, but most of the gallbladder epithelium was intact (fig 2B). Tumour invasion was seen in the cystic duct, common bile duct, portal vein, part of the liver parenchyma, hepatoduodenal ligament, omentum, part of the muscular layer of the transverse colon, and duodenum. The histological differential diagnosis for such small round cell tumours included undifferentiated carcinoma, small cell carcinoma, Ewing's sarcoma, rhabdomyosarcoma, monophasic synovial sarcoma, nephroblastoma, and haemopoietic tumour.

Upon immunohistochemical examination, the tumour cells showed diffuse and strong reactivity for myeloperoxidase (MPO), CD43, and c-kit protein (CD117) (fig 2A-C), and weak reactivity for CD45 (LCA) and CD99. The cells were negative for CD20, CD79a, CD3, CD34, CD45RO (UCHL-1), CD68, CD56, terminal deoxynucleotidyl transferase, WT1, desmin, vimentin, and cytokeratins (CAM 5.2, AE 1/3, and KL1). Based on these results, we made a final diagnosis of EMMT.

After surgery, the patient underwent combination chemotherapy as prescribed for cases of acute myeloblastic leukaemia, but clinical investigations-including computerised tomography and a bone marrow trephine biopsy

Abbreviations: EMMET, extramedullary myeloid tumour; MPO, myeloperoxidase 

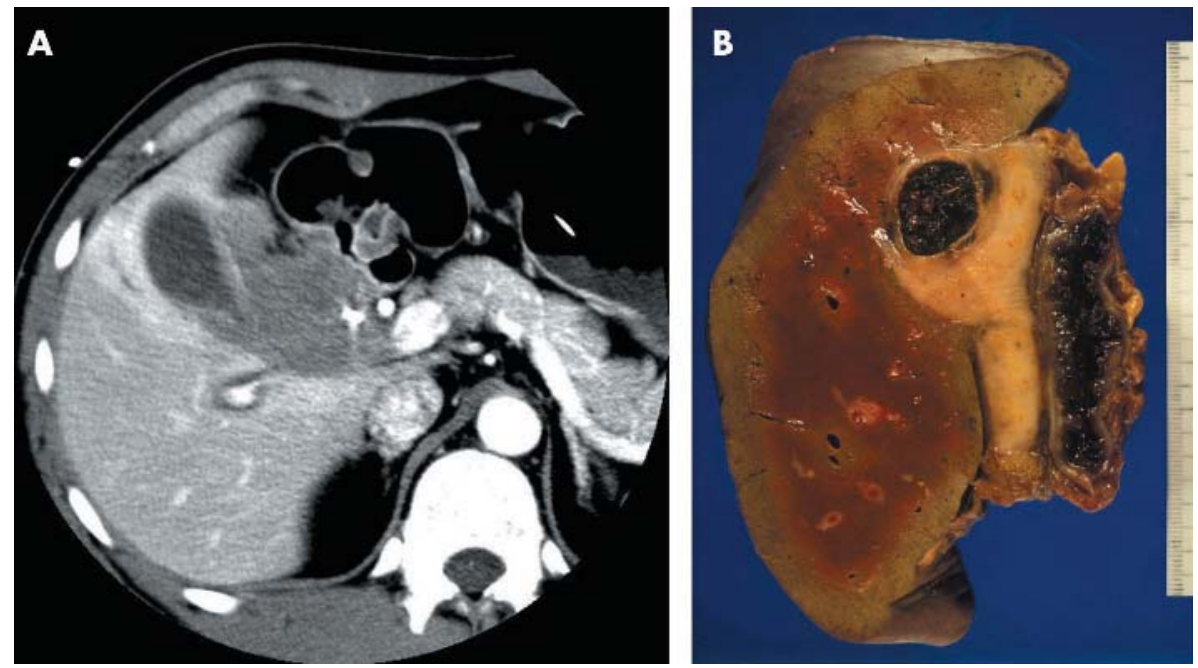

Figure 1 (A) Abdominal computerised tomography image showing partial infiltration of the tumour into the gallbladder wall but no sign of invasion of the liver or portal vein. (B) Macroscopically, the gallbladder lumen is filled with blood and degenerative tissue, and the cut surface of the tumour has a nodular, well circumscribed, glistening appearance.

specimen-did not detect more lesions. The patient did not develop acute leukaemia during a follow up period of four years.

\section{DISCUSSION}

A large proportion (75-86\%) of EMMTs in non-leukaemic patients are initially misdiagnosed ${ }^{2}$ because of their morphological and immunohistochemical similarity to other small round cell tumours. Most of them are diagnosed as malignant lymphoma, and occasionally as Ewing's sarcoma or eosinophilic granuloma. ${ }^{4}$

"In cases of suspected extramedullary myeloid tumour, antibodies to myeloperoxidase and CD43 should be used, along with other B cell and T cell specific antibodies"
Some small round cell tumours, such as undifferentiated carcinoma and small cell carcinoma, usually show aggressive invasion, but in our case the lesion had not invaded the portal vein or the gallbladder epithelium, and staining for cytokeratins was negative. Other small round cell tumours, such as Ewing's sarcoma, rhabdomyosarcoma, monophasic synovial sarcoma, and nephroblastoma, were also eliminated because of negative immunostaining for desmin, cytokeratins, and WTl, respectively. Malignant lymphomas may be positive for CD45 (LCA) along with either pan B cell (CD79a, L-26) or T cell (CD3) markers, but are negative for MPO, which was positive in our case.

Immunohistochemical markers, such as CD43 and MPO, may be helpful in the diagnosis of EMMT. However, a few cases of EMMT show reactivity for pan B cell (CD79a, L-26) or T cell (CD3) markers. Furthermore, CD43 is an excellent

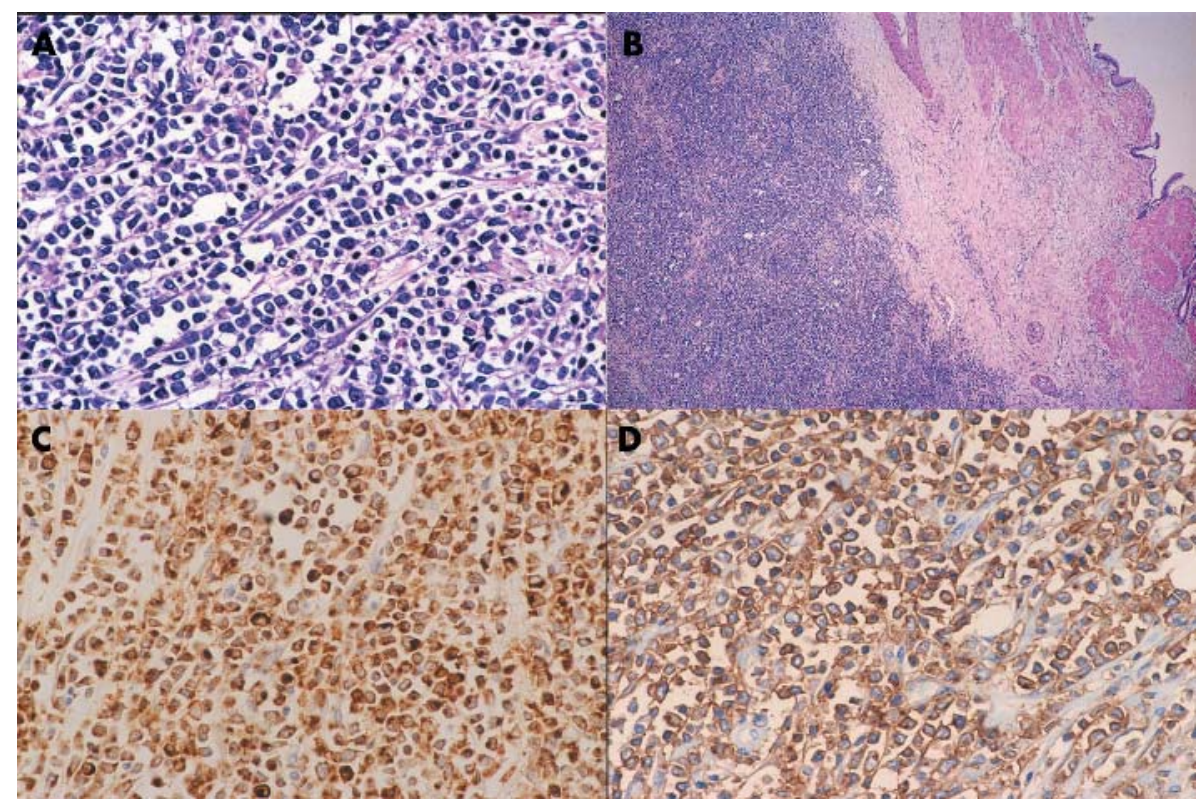

Figure 2 (A) The tumour cells have various amounts of eosinophilic cytoplasm and are arranged in a trabecular to sheet-like pattern (haematoxylin and eosin (H\&E) stain; original magnification, $\times 400)$. (B) Most of the gallbladder epithelium is not involved (H\&E stain; original magnification, $\times 40$ ). The fumour cells show diffuse and strong reactivity for (C) myeloperoxidase and (D) CD43 (original magnification, $\times 400$ ). 


\section{Take home messages}

- We present a rare case of extramedullary myeloid tumour of the gallbladder in a non-leukaemic patient that was diagnosed successfully using an appropriate panel of immunohistochemical stains

- The patient underwent combination chemotherapy after diagnosis and has not developed leukaemia after four years of follow up

- It is important not to misdiagnose such cases as malignant lymphoma, because the pathological diagnosis influences the prognosis of the patient

marker for myeloid cells and is also used as a T cell marker. ${ }^{2}$ If myeloid associated antigens are not investigated, a misdiagnosis of malignant lymphoma is likely. Therefore, in cases of suspected EMMT, antibodies to MPO and CD43 should be used, along with other $\mathrm{B}$ cell and $\mathrm{T}$ cell specific antibodies.

Recently, c-kit tyrosine kinase inhibitors (such as Glivec) have been studied as possible treatments for haemopoietic malignancies, so that c-kit detection may have important implications for treatment. Jian et al reported c-kit reactivity in $87 \%$ of EMMT cases, ${ }^{5}$ and many tumours have been shown to express CD117/KIT as assessed by the anti-KIT polyclonal antibody (DakoCytomation, A4502; Glostrup, Denmark) used in our laboratory, which has low specificity. Although tumours carrying mutations of either c-kit or the platelet derived growth factor $\alpha$ gene (for example, gastrointestinal stromal tumours) are sensitive to tyrosine kinase inhibitors, in the absence of an accompanying mutation other KIT positive tumours show no therapeutic benefit with Glivec. ${ }^{6}$ We were unable to isolate and sequence the c-kit gene from formalin fixed, paraffin wax embedded tissue sections of our present case.

Most non-leukaemic patients with EMMT develop acute leukaemia one to 49 months after the diagnosis (mean, 10.5). ${ }^{1}$ If the case is correctly diagnosed as EMMT at the initial presentation, and the patient receives intensive systemic chemotherapy with or without radiotherapy, longterm survival is good. ${ }^{7}$ Aggressive forms of treatment are necessary for patients who present with acute myeloid leukaemia after being treated with chemotherapy for nonHodgkin lymphoma as a result of initial misdiagnosis. These patients frequently fail to attain durable remission and have a poor prognosis. ${ }^{2}$

In summary, we present a rare case of EMMT of the gallbladder in a non-leukaemic patient that was diagnosed successfully using an appropriate panel of immunohistochemical markers. Care should be taken not to misdiagnose such cases as malignant lymphoma, because the pathological diagnosis influences the prognosis of the patient.

\section{Authors' affiliations}

H Ojima, T Hasegawa, Y Matsuno, Pathology Division, National Cancer Centre Research Institute and Hospital, 5-1-1 Tsukiii, Chuo-ku, Tokyo 101-0045, Japan

M Sakamoto, Department of Pathology, Keio University School of Medicine, Tokyo 160-0016, Japan

Correspondence to: Dr T Hasegawa, Pathology Division, National Cancer Centre Research Institute, 5-1-1 Tsukiii, Chuo-ku,

Tokyo 101-0045, Japan; tdhasega@ ncc.go.jp

Accepted for publication 18 August 2004

\section{REFERENCES}

1 Neiman RS, Barcos M, Berard C, et al. Granulocytic sarcoma: a clinicopathologic study of 61 biopsied cases. Cancer 1981;48:1426-37.

2 Menasce LP, Banerjee SS, Beckett E, et al. Extra-medullary myeloid tumor (granulocytic sarcoma) is often misdiagnosed: a study of 26 cases. Histopathology 1999;34:391-8.

3 Geddy PM, Wedgwood KR. Myelofibrosis presenting as chronic cholecystitis. J Clin Pathol 1996;49:428-9.

4 Hutchison RE, Kurec AS, Davey FR. Granulocytic sarcoma. Clin Lab Med 1990;10:889-901.

5 Jian C, Rudolph R, Yanuck III, et al. c-kit (CD 1 17) reactivity in extramedullary myeloid tumor/granulocytic sarcoma. Arch Pathol Lab Med 2001; 125:1448-52.

6 Yamaguchi U, Hasegawa T, Masuda T, et al. Differential diagnosis of gastrointestinal stromal tumor and other spindle cell tumors in the gastrointestinal tract based on immunohistochemical analysis. Virchows Arch 2004;445: 142-50.

7 Oliva E, Ferry JA, Young RH, et al. Granulocytic sarcoma of the female genital tract: a clinicopathologic study of 11 cases. Am J Surg Pathol 1997;21:1156-65. 